\title{
Kernos
}

Revue internationale et pluridisciplinaire de religion grecque antique

$10 \mid 1997$

Varia

\section{M.-M. MACTOUX, E. GENY (éds), Discours religieux dans l'Antiquité}

\section{André Motte}

URL : http://journals.openedition.org/kernos/685

DOI : $10.4000 /$ kernos.685

ISSN : 2034-7871

\section{Éditeur}

Centre international d'étude de la religion grecque antique

\section{Édition imprimée}

Date de publication : 1 janvier 1997

Pagination : 348-350

ISSN : 0776-3824

Référence électronique

André Motte, « M.-M. mactoux, E. geny (éds), Discours religieux dans l'Antiquité », Kernos [En ligne], 10 | 1997, mis en ligne le 12 avril 2011, consulté le 24 septembre 2020. URL : http:// journals.openedition.org/kernos/685; DOI : https://doi.org/10.4000/kernos.685 
Cet ouvrage, qui enrichit de façon originale notre connaissance des Nuées, se termine par une bibliographie sélective, forte d'environ quatre-vingt titres.

André MOTTE

(Université de Liège)

Joachim Lacrosse, L'amour chez Plotin. Erôs hénologique, Erôs noétique, Erôs psychique, Bruxelles, Éditions Ousia, 1994. 1 vol. $14 \times 21 \mathrm{~cm}$, 143 p. (Cabiers de pbilosopbie ancienne). ISBN : 2-87060-044-5.

Il existait déjà, sur la figure d'Éros chez Plotin, une littérature assez abondante, mais on se réjouira de voir ici le thème exploré d'une façon systématique qui permet à la fois d'en aborder les multiples facettes et de les replacer ensuite dans une perspective globale. La première partie est opportunément consacrée à l'exégèse des données platoniciennes exploitées par le lointain disciple; continuités et ruptures y sont bien mises en évidence, ce qui constitue une excellente introduction aux différents thèmes plotiniens examinés dans la suite. Dans le second chapitre, l'érôs est étudié dans ses rapports avec la conversion de l'âme et avec celle du Nô̂s vers l'Un; l'occasion se présente ici d'aborder la démonologie de Plotin, - Érôs est identifié au « Grand Démon »-, et sa morale sexuelle. Le dernier chapitre examine un aspect moins connu du thème, bien que le plus original et le plus fondamental, celui de l'Érôs hénologique, l'amour de soi par lequel l'Un engendre, sans en être altéré, les réalités issues de lui. C'est ici que l'on perçoit notamment combien l'érôs et le logos sont très étroitement associés. Une synthèse éclairante est fournie dans la conclusion justement intitulée «L'omniprésence de l'amour ». Importante bibliographie.

André MOTTE

(Université de Liège)

Marie-Madeleine Mactoux, Evelyne GeNy (éds), Discours religieux dans l'Antiquité. Actes du colloque (Besançon, 27-28 janvier 1995), Paris, Les Belles Lettres, 1995. 1 vol. 15,5 ×24 cm, 322 p. (Annales littéraires de l'Université de Besançon, 578. Centre de Recherches d'Histoire ancienne, 150). ISBN : 2-251-60578-9.

Les 15 communications que reproduisent ces Actes couvrent une vaste période qui va d'Hésiode à Vincent de Lérins, un auteur chrétien du $v^{\mathrm{e}}$ siècle, et la thématique abordée, tant en ce qui concerne les types de discours religieux pris en compte que les analyses auxquelles on les soumet, présente à son tour une grande diversité. Aussi n'est-il pas inutile de prendre d'abord connaissance de l'avant-propos synthétique, dû à M.-M. MACTouX, avant d'entamer la lecture de l'ouvrage; y sont notamment présentées les trois pistes de réflexion qui ont servi à regrouper les interventions. La première est centrée sur les « destinataires et la réception des discours religieux inscrits dans les discours eux-mêmes sous diverses modalités ». Une contribution de Cl. CALAME (Invocations et commentaires "orpbiques": transpositions funéraires de discours religieux) ouvre cette série; riche d'érudition et d'observations minutieuses, mais d'une lecture difficile, elle confronte, dans leurs fonctions énonciative et performative, deux types de discours "mystiques " et à usage funéraire, le papyrus de Dervéni et les deux lamelles d'or récemment découvertes à Pélinna. J.-M. PAILler 
(Dionysos entre la vue, le cri et l'écrit. Y a-t-il un discours religieux dionysiaque) s'attache à montrer que, malgré la place importante qu'occupent la vue et le cri dans les manifestations de la religion dionysiaque, le dieu s'identifie, de façon persistante, « au pouvoir transformateur d'un langage consacré » qui lui permet de renaître d'âge en âge. Dans un article particulièrement copieux (Discours et conversion cbez Clément d'Alexandrie), C. DAude étudie les deux types de discours dont use le Pédagogue, l'un qui, évoquant la vie quotidienne, se veut réaliste, l'autre dont la portée est religieuse et qui, interprétant ces réalités, exerce sur le lecteur « une contrainte permanente en vue de sa conversion ». Le sujet choisi par P. SCARPI (Récit mythique et saecularia litteratura) concerne lui aussi le débat entre christianisme et monde antique, mais cette fois chez un auteur latin, Tertullien; l'apologiste chrétien, dont l'effort sera poursuivi par Augustin, entreprend de disqualifier la religion païenne en reléguant le mythe dans l'espace du ludus; à noter, précédant cette brève démonstration, quelques pages sur " le débat autour du mythe en Grèce ». Cette première série se termine par une intéressante contribution de C. JOURDAIN-ANNEQUIN (De l'exploit béroíque à la biograpbie) illustrant les principaux avatars qu'a connus le traitement de la figure de l'Héraclès grec.

La deuxième piste de recherches concerne l'interdiscours religieux. M.-Chr. LECLERC (Poésie et religion chez Hésiode) fait voir la variété du discours religieux chez ce poète, et aussi sa plasticité qui, de la Théogonie aux Travaux, entraîne des écarts significatifs. Soumettant à une étude comparative les mythes d'Athéna et d'Hermès, Cl. LEDUC conclut que le mythe peut, comme c'est ici le cas, donner à voir - et à savoir - ce qu'est la divinité par le truchement de signes énigmatiques; cette révélation indicible que véhicule le récit de la geste divine porte peut-être la trace d'une «structure d'initiation ". Un intéressant exemple d'appropriation et de transformation du mythe grec dans une culture étrangère est présenté par F. SIEGERT (L'Héraclès des Juifs). La contribution suivante s'éloigne davantage du domaine de la religion grecque (J.D. Dubors, Vie de Jésus et vie de Mani au cœur des débats entre cbrétiens ortbodoxes et manichéens), et c'est par un titre très explicite que se clôture la série (N. FIcK, Saint Augustin pourfendeur des démons païens ou la critique de la démonologie d'Apulée, De Civ. Dei, VIII, 14-22).

Retour à la religion grecque dans les trois premiers articles que présente la troisième partie de l'ouvrage intitulée «Le codage politique ». P. Brulé (La liste des premiers rois d'Atbènes dans la Bibliothèque d'Apollodore. Histoire et politique) aborde l'étude de cette généalogie royale en adoptant une perspective qui privilégie l'examen de la lignée par rapport à celui des « règnes » qui sont comme les maillons de la chaîne; au centre du discours, la trame généalogique n'en structure pas seulement la forme, elle en explicite aussi le fond; plusieurs tableaux accompagnent l'analyse. Non moins intéressant est l'essai de P. VGELKE (Théâtre, religion, politique. Le drame sayrique et les réformes de Clisthènes) qui explore la relation entre l'espace représenté au théâtre par le drame satyrique et l'espace de la cité que réorganisent les réformes de Clisthènes, en y joignant un troisième terme, l'espace de la cité tel qu'il se constitue dans le culte de Dionysos Eleutbereus. Très substantielle, la contribution de M.-M. MACTOUX (Une argumentation barbare (Hérodote, II, 54-57)) analyse la stratégie argumentative qui se fait jour dans ce passage relatif aux origines de l'oracle de Dodone et montre qu'elle se fonde sur l'adhésion idéologique d'un auditoire dont elle entend rappeler la cohésion civique. Citons encore, pour être complet, les deux 
derniers articles : le premier (E. SMADJA, Statue, image et culte de l'empereur en Afrique) est consacré à un type de discours iconique, le second (R. NOUALHAT, Analyse d'un discours théologique dogmatique : le Commonitorium de Vincent de Lérins) s'appuie sur un traitement informatique du texte.

Ce bref aperçu met en évidence, - trop facilement -, le caractère assez disparate des études rassemblées, il n'en montre pas assez l'intérêt, souvent lié à la nouveauté des démarches suivies.

A. MOTTE

(Université de Liège)

Jean-Michel Adam, Marie-Jeanne Borel, Claude Calame, Mondher Kilani, Le discours antbropologique. Description, narration, savoir, Lausanne, Éditions Payot Sciences humaines, 1995, 2e éd. revue et augmentée. 1 vol. $14 \times 22,5 \mathrm{~cm}, 285$ p. ISBN : 2-601-03169-7.

Le thème de cet ouvrage est la connaissance anthropologique, signe d'un temps où la discipline anthropologique s'interroge sur son propre statut et sur son rapport au savoir. Description et narration sont au cœur du travail de l'anthropologue qui, «non seulement a pour champ d'investigation les cultures des autres, mais [doit] être conscient que ses propres procédures de schématisation et d'écriture sont elles-mêmes soumises à ces processus, qui relèvent notamment de la métaphore » (p. 6). Il s'agira dès lors de soumettre le discours anthropologique à une critique centrée sur ses propres marques et ses modalités d'énonciation particulières. Le processus qui « mène de l'expérience individuelle de terrain à la production de la connaissance anthropologique générale » (p. 7) est au centre des préoccupations actuelles qui concernent aussi, en fonction de leurs spécificités, la sociologie et l'histoire.

Depuis plusieurs décennies, l'anthropologie culturelle et sociale s'est largement appliquée au domaine de l'antiquité, en fonction de traditions nationales particulières. Toutefois, le matériau de base n'est pas l'enquête de terrain, mais des discours produits par les Anciens dans des perspectives bien différentes de celles de l'anthropologue contemporain. C'est de ce type de particularité que discute Claude Calame dans ce livre, aux côtés de trois de ses collègues de l'Université de Lausanne, respectivement linguiste, logicienne-épistémologue, anthropologue. La contribution sur la Grèce comprend deux volets. Une première approche analyse et évalue le discours contemporain de type anthropologique sur la religion grecque, en l'occurrence les interprétations des Oschophories et du personnage de Thésée dans l'œuvre de Jane E. Harrison (Du figuratif au thématique: aspects narratifs et interprétatifs de la description en anthropologie de la Grèce ancienne, p. 101-120). La deuxième approche, par le biais de l'analyse de la description du sanctuaire d'Héra à Olympie par Pausanias, traite de la démarche " anthropologique » du Périégète et des matériaux qu'il met ainsi à la disposition de notre propre analyse - qu'elle soit ou non « anthropologique » (Pausanias le Périégète en etbnographe ou comment décrire un culte grec, p. 205-226). Et Claude Calame de montrer combien la reformulation du discours de Pausanias sur l'Héra d'Olympie dans les travaux modernes (Weniger en 1883, lui-même en 1977 et Detienne en 1986) efface les procédures énonciatives du texte ancien au profit de son exploitation comme "réalité ». Le discours de l'historien de la religion grecque deviendrait alors "illusionniste ", tendant à prendre ses reconstructions pour des réalités (que ce soient 